\title{
HANDGRIP STRENGTH EVALUATION USING NEURO FUZZY APPROACH
}

\author{
Woo Chaw Seng, Mahsa Chitsaz \\ Department of Artificial Intelligence, \\ Faculty of Computer Science and Information Technology, \\ University of Malaya, Kuala Lumpur, Malaysia. \\ Email: cswoo@um.edu.my,mchitsaz@perdana.um.edu.my
}

\begin{abstract}
Handgrip assessment is a useful method to monitor patient rehabilitation. The neurofuzzy analysis provides system identification and interpretability of fuzzy models and learning capability of neural networks. The purpose of this study is to collect handgrip strength of patients and distinguish them from the normal persons. Multilevel Perception neural network utilizes the back-propagation learning algorithm is suitable to discover relationships and patterns in the dataset. When the parameters are well tuned, the expert rules in the training data are captured and stored as expert weights of the neural network. The expert rules define the membership function for the fuzzy system. The fuzzy model based on the membership function, fed in by the neural network will intelligently classify the data. The results indicate that the classification accuracy of normal and pathological patients are $90 \%$ and $75 \%$ respectively. Moreover, this research demonstrates the feasibility of a novel handgrip design because the force measurements variance of the conventional LIDO machine and our designed handgrip is only 0.169 .
\end{abstract}

Keywords: Handgrip strength evaluation, Neuro-Fuzzy system, Fuzzy Logic, Neural network.

\section{INTRODUCTION}

Handgrip assessment is a useful method to monitor patient rehabilitation $[1,2,3]$. Besides, it is used to investigate certain diseases, old age disability, and hand disability, morbidity, and mortality prediction, prognostic indicator in surgical patient and feigning effort of patient with secondary gain [3]. A standard handgrip assessment device adopts the principle of force produced by a handgrip, which is converted into electrical signal for analysis. Each method of assessment has been employed various devices that have their owned limitations.

The designed system composes of two parts; the mechanical handgrip system and the signal acquisition processing system. The mechanical handgrip system is designed based on nutcracker model. Moreover, it is made from aluminum, which is strong, light and durable[4, 5]. Strain gauges are used to detect forces acting on the gripper. The output from the sensors are amplified by the strain gauge amplifier and preconditioning circuits. Isometric force from the hand is applied to the two handles of the grip. It is transferred as electrical signal by the strain sensors that are placed in a ring between the handles. The electrical voltage signal from the sensors are pre-processed and amplified before connecting it to a personal computer equipped with a data acquisition system. The mean, maximum and minimum of the acquired signal of force are calculated, and then will be stored in the database in order to be compared with the available values for diagnosis. The classification computation is based on Adaptive Neuro Fuzzy Inference System (ANFIS) [6]. ANFIS is a fuzzy inference system implemented in framework of adaptive neural networks. Input/output datasets are used to construct a fuzzy inference system whose membership functions are tuned using either a learning algorithm or an expert to specify a fuzzy inference. Finally, the system is trained with the data pairs by an adaptive network [7].

In this section, we present a short description of Fuzzy logic, Artificial Neural Network (ANN) and Neuro-Fuzzy. Section 2 is a brief summary of recent researches using Neuro-Fuzzy in medical field. Section 3 gives the details of the approach and explicates algorithms used in our work. Section 4 analyses the experimental results and section 5 discusses the results. Finally, Section 6 concludes our work. 


\subsection{FUZZY LOGIC}

Fuzzy logic is a method of rule-based decision-making used for expert systems and process control that emulates the rule-of-thumbs thought process human beings use [8]. Fuzzy set is different from traditional Boolean set theory. Fuzzy set theory allows for partial membership, which might be any value between 0 and 1 . In this logic, the propositions can be represented with degrees of truthfulness and falsehood. In fuzzy logic method, the rule-based corresponding membership function is used to analyze the input and determine the output [9]. Fuzzy logic is a logic that presents how the human brain categorizes objects, evaluates conditions and process decisions [9]. A paradigm is a set of rules and regulation which defines boundaries and tell us what to do to be successful in solving problems within these boundaries. From a theoretical point of view, a fuzzy set can be interpreted as a possibility distribution presenting a linguistic variable along its universe of discourse. Let ${ }^{X}$ be any element of the universe discourse $U$, and a fuzzy set $F$ defined $U$ can be written as:

$$
\begin{array}{ll}
F=\left\{\left(x_{,} \mu_{F}(x)\right) \mid x \in U\right\} & \\
F=\int_{x} \mu_{F}(x) / x & \text { if } U \text { is continuous, } \\
\mathrm{F}=\sum_{\mathrm{i}=1}^{\mathrm{n}} \mu_{F}\left(x_{i}\right) / x_{i} & \text { if } U \text { is discrete. }
\end{array}
$$

Where $\mu_{F}(\quad)$ is the membership function, which describes the characteristics of fuzzy set $F$.

There are several shapes of fuzzy sets that are commonly used such as triangular, Gaussian, trapezoidal, bell shape, etc. Three types of input space partitioning methods are generally used which are grid, tree and scatter partition as shown in Fig. 1 [10].

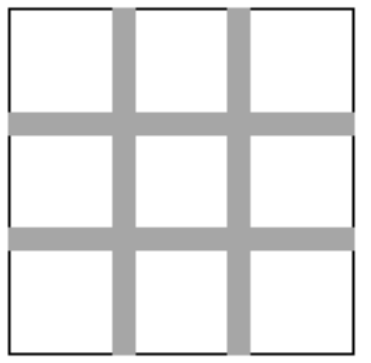

(a)

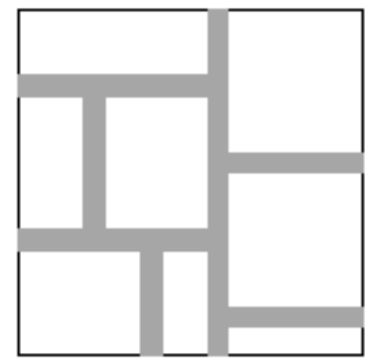

(b)

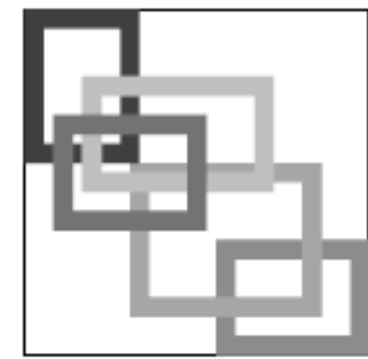

(c)

Fig. 1 Three Methods of Input Space Partitioning for Fuzzy Systems: (a) Grid Partition; (b) Tree Partition; (c) Scatter Partition [10]

The imprecise modes of fuzzy reasoning offers some advantages in control systems such as simplicity and structural procedures during the design stage and with a shorter design cycle time. Besides, fuzzy control offers some practical advantages during real time implementation such as saving in both installation and production costs, easy to maintain and making alteration, improved energy efficiency compared to conventional control methods and providing excellent performance.

\subsection{ARTIFICIAL NEURAL NETWORK}

A neural network is a paralleled distributed processor consists of simple processing units with a natural propensity for storing experiential knowledge and making it available for use [11]. Neural network resembles the two factors of brain in recognition. Knowledge is the first factor that is acquired by the network from its environment through a learning process. Then, inter-neuron connection strengths known as synaptic weights are used to store the acquired knowledge. Artificial neural networks (ANN) are algorithms that imitate the functions of biological nervous systems of living creatures. It consists of a number of neurons in parallel way with each of the neuron processing its own 
information locally. The firing strength of the signal is determined by an activation function that is associated with the neuron.

Based on structural configuration, ANN can be classified into three categories: feedforward, feedback and cellular networks. In feedforward ANN, the signal processing flows only in a forward direction within its architecture. In feedback architecture, outputs from the neurons are being fed back to the inputs of the neurons at the same or following layer.

There are three categories of ANN learning models based on learning methodologies: supervised, unsupervised and reinforcement learning [12]. Supervised learning needs to be supplied with example input-output sample. Adversely, unsupervised training usually performed through competitive learning where the neurons compete with each other to become the winner of a particular input vector. The reinforcement learning algorithm performs the tuning procedures based on a feedback critic signal that is deducted from its environment.

A trained neural network can be thought of an 'expert' that can be used to provide prediction of new situations. The advantages of ANN are adaptive learning, self-organization, real time operation and fault tolerance via redundant information coding [13].

\subsection{INTEGRATION OF NEURO-FUZZY}

Every intelligent technique has specific computational properties that make them suited for particular problems and not for the others. For example, neural networks are appropriate for recognizing patterns, but it is not useful in explaining how they reach their decisions. In addition, Fuzzy Logic Systems (FLS) are suitable for explaining their decision but they are not promising in automatic acquisition of rules. These limitations motivated researchers to create intelligent hybrid systems where two or more techniques are combined to overcome the individual limitations. The low-level learning and computational power of ANN can be injected into FLS, while high-level, and systematic reasoning rule of FLS can be embedded into the ANN structures. Thus, more transparency can be obtained for ANN while the FLS allows automatic tuning of parameters.

Based on the functionality modification of ANN, the combination of FLS and ANN generally can be classified into two categories: fuzzy network system and fuzzified network systems. In neurofuzzy type of fuzzy network systems, the FLS is installed in an isomorphic architecture of ANN. On the other hand, the integration retains the basic properties and architectures of neural networks in fuzzified network systems.

Neurofuzzy system aims to provide FLS with a typical ANN to augment numerical processing of fuzzy sets such as membership function elicitation to draw out and realization of mapping between fuzzy sets and fuzzy rules. This synergetic integration reaps the advantage of both ANN and FLS. The structure of neural networks provides the learning and adaption capability to FLS. Meanwhile, FLS provides ANN with a structural framework comprises of high-level rules. ANN is most widely diffused with FLS. It has three or five-layer of feed forward neural networks, while the most widely applied learning algorithm is the back propagation method [10, 14]. Each layer serves input linguistic nodes, input term nodes, rule nodes, output term nodes and output linguistic nodes. The function of each layer is mapped directly into the FLS. Moreover, Hunt and Hass [11] proposed a fuzzy neural model based on functionality equivalence of a takagi-sugeno fuzzy inference model and a B-spline basis function neural network. The proposed network possesses one interesting property where the basis function form a partition of unity thus the input to the network activates every possible combination of active basis functions.

\section{RELATED WORK}

A number of researches have been recently done to reduce the cost and performance of devices in various medical and engineering applications. However there is no research in handgrip strength assessment to analyze the outcome of force value using hybrid models. In the following paragraphs, we mention a few researches that use handgrip tool to measure the force value for different purposes. 
McGory [15] proposed a system to provide accurate and repeatable measurement of mean (overall) grip force, estimation of the magnitude and direction of the grip force vector, and the simultaneous determination of applied moments produced in a task simulation. The proposed hand tool analysis system composed of four major components: an end effecter; a sensor for measuring reactive moments applied at the end effecter; a grip force sensing core (grip core); and a handle. Handles of various sizes and shapes can be mounted to the grip core. The moment sensing end of the handle has a fitting to accept various end effecters. The result shows that the error in localization decayed exponentially with increasing load. Errors of $3 \mathrm{~mm}$ or less were observed with loads exceeding $25 \mathrm{~N}$. Moreover, the results of the general linear model analysis indicated that grip force and applied moment were significantly greater for the high-precision than for the low-precision tasks during the initial phase $(25 \%)$ of the cutting replications at both the thick and thin levels of clay thickness.

Hoozemans and Dieen [16] invented a standardized gripping tool to calibrate the measurement with a series of dynamic bursts up to $300 \mathrm{~N}$ resulted in a valid prediction of grip forces based on the normalized electromyography (EMG) of 6 forearm muscles. Handgrip forces were measured using a custom-made strain gauge based force transducer. Two strain gauges were built within the handle just above and beneath the hand and both measured the force applied by the fingers and opposed by the thumb. The calibration measurement with steps of static grip force generally showed regression coefficients furthest from $1.0(0.67,0.74$, and 0.82 for grip widths 59,67 and $75 \mathrm{~mm}$ respectively), the lowest variance explained $(54 \%, 69 \%$, and $78 \%$ ) and the highest values of the mean absolute difference between the observed and predicted forces (80, 62 and $52 \mathrm{~N}$ ). Absolute differences between observed and predicted grip forces were small, which shows that the proposed method might be used for the ergonomic evaluation of the use of hand tools.

As mentioned in this section, the designed devices are implemented to measure the force value of handgrip strength. However, none of them have the feature of storing data to post-process it for treatment planning, diagnosis and prognosis study purposes.

\section{METHODOLOGY}

Our Handgrip system is the combination of hardware and software, which embedded with neurofuzzy applications, as shown in Fig. 2. The hardware component includes the mechanical development and the electronic development. It briefly consists of the gripper, strain detector and electronic circuit. In software development, the signal analyzing is including filtering, averaging, amplification and recording the data.

Hardware

Software

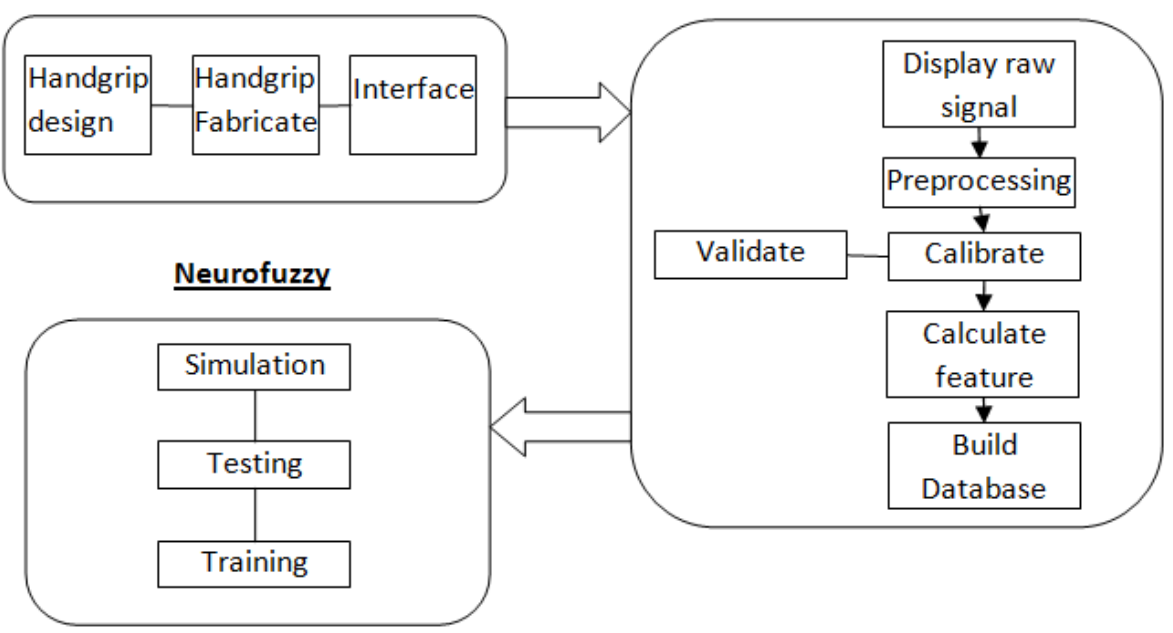

Fig. 2: The Overall View of the Proposed Method

\subsection{HARDWARE DESIGN}

The mechanical handgrip system is developed based on the nutcracker model. The gripper consists of three parts, i.e. hand, neck and holder. Isometric force from the hand is applied to two handles of the gripper. The head is used 
to fit in the ring component. The ring placed on the centre for maximum detection and efficiency. The designed handgrip is shown in Fig. 3.

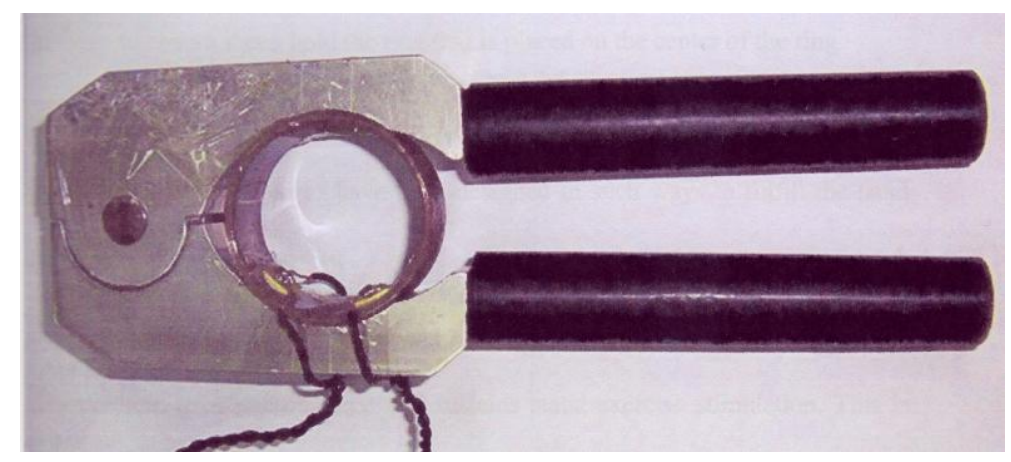

Fig. 3 The Designed Handgrip

According to mechanical features, the handgrip is presented as a hand-held device for isometric force measurements, which is simple to use. A piece of soft cover has been attached to the place for hands to make it comfortable for patients. Moreover, the holder is adjustable to make it easy for different hand sizes in our design. The force principle is used for a strain calculation:

$$
\hat{\Gamma}=r . F
$$

Where $r$ is the vertical distance between applied force and rotational axis. $F$ is defined as the force applied to the system. To measure strain $(\varepsilon)$, typically two or four strain gages are applied in Wheatstone bridge in one of several bridge configurations. The bridge connection can be Half Wave Bridge (specifically for two strain gages) or Full Wave Bridge (for complete four strain gages). Strain is measured from the deformation ring by using a strain gage. The load detector senses changes in electrical resistance that varies in proportion to the amount of strain in the device as shown in Fig. 4.

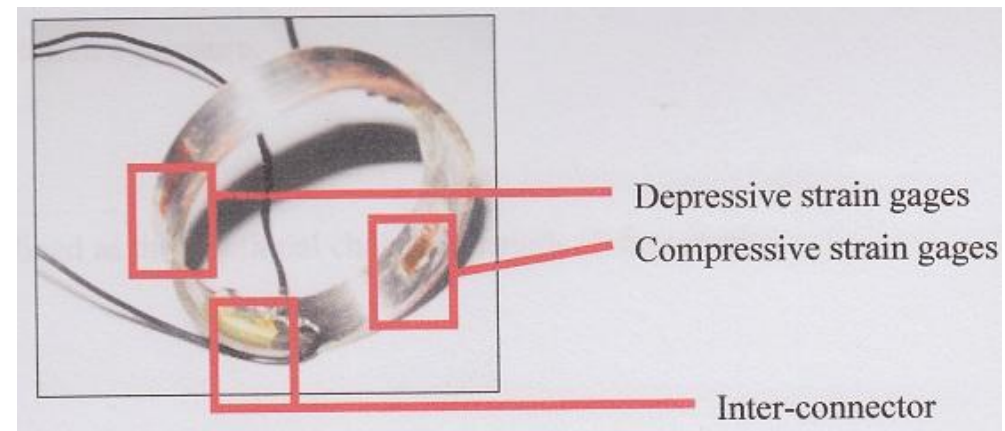

Fig. 4 Ring with Couple of Strain Gages

Strain measurements rarely involve quantities larger than few micro-strains. Therefore, measuring strain requires accurate measurements of very small changes in resistance. We used the Wheatstone bridge to change the strain to voltage. Strain gage resistance will unbalance the bridge and produce a nonzero output voltage when changes occurred in the system. 


\subsection{SOFTWARE ARCHITECTURE}

The hardware has to communicate with a computer system for data analysis. The USB DAQ (Data Acquisition Card) is used to enable communication between the handgrip and the computer system. The DAQ becomes the interface between the computer and the outside world. Its primary function is to convert the analog signal to digital signal, and feed it into the computer for further processing. The software architecture mainly consisted of data acquisition and analysis as shown in Fig. 5. The raw data are analog signals that are converted to digital data by the DAQ card. The four strain gauges are configured in a Wheatstone bridge formation in order to balance any errors that may arise due to the fluctuation of supply voltage, changes in temperature and any residual resistance or inductance that may occur from the lead wires and contact resistance. The processing of data is divided into two modules of data filtering and data averaging. The signal acquired using the DAQ card usually contains noise. The noise originated from either the hardware, connections between the circuits or stray electromagnetic signal. Since the noise value is usually in high frequency the cut off frequency of $10 \mathrm{~Hz}$ is sufficient to filter out the noise. A low pass Butterworth filter of order 3, effectively removes the noise and the signal can be recovered. Finally, the analyzed data depicts the maximum and minimum value of signal frequency. These information will use in neurofuzzy module.

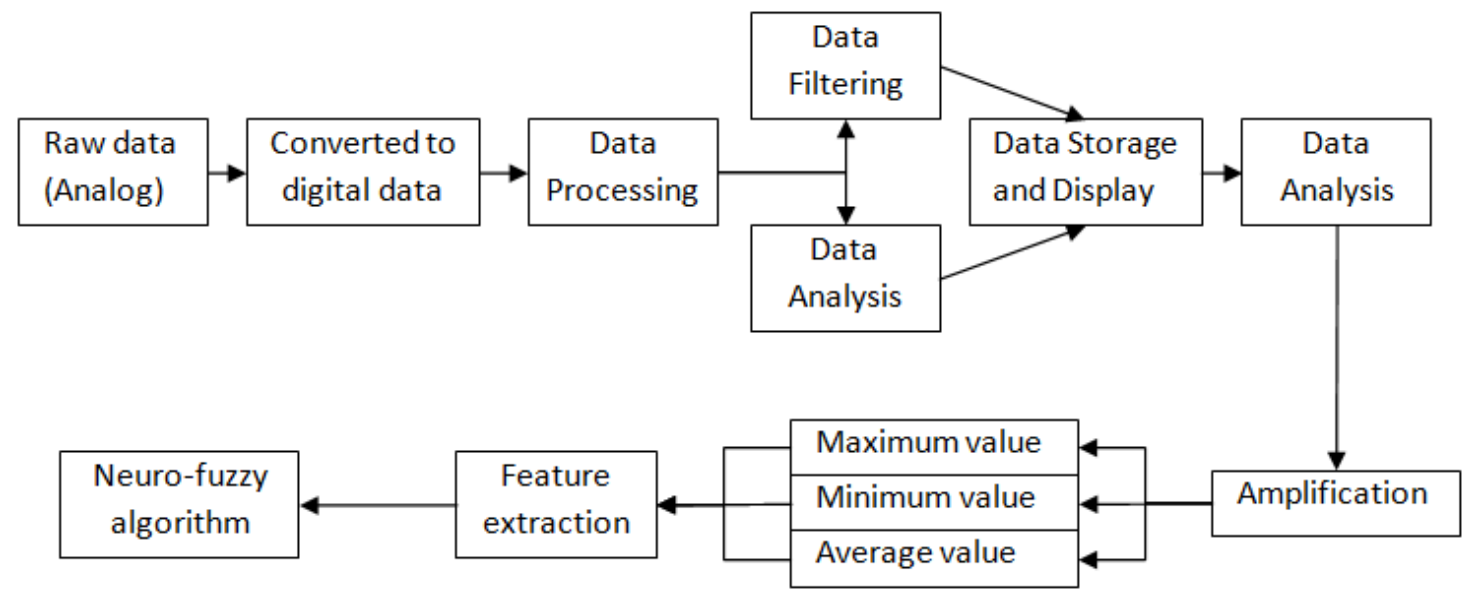

Fig. 5 Software Architecture

\subsection{NEURO-FUZZY MODULE}

The neurofuzzy module is the main engine, which intelligently processes the preprocessed data. The input data is age, race, sex, height, weight and handgrip tension (force). Multi-Level Perception (MLP) utilizes the backpropagation learning algorithm that is suitable to discover relationships and patterns in the dataset. The MLP architecture consists of two layers, an input layer of six neurons and an output unit of one neuron. A set of 600 data consists of six fields was obtained, 400 data set randomly chosen to train the MLP, another 200 data were used for testing and validation. When the parameter are well set and well tuned, the invariant patterns, the dynamic present and expert rules in the training data are captured and intrinsically stored as expert weights of the neural network. The expert rules stores as expert weights that can be used to define the membership function for the fuzzy system. The fuzzy model based on the membership function, fed in by the neural network will intelligently classify the data. The trained neurofuzzy model was tested with 100 set of data and verified. In a conventional fuzzy inference system, an expert who is familiar with the target system determines the numbers of rules. In this research, the data is too complex , hence we utilize a front-end processor to generate the membership function automatically. To initialize the FIS using ANFIS, the subtractive clustering is chosen because the numbers of clusters are unknown for a given set of data. The task of the learning algorithm for this architecture is to tune all modifiable parameters in order to make the ANFIS output match the training data. When the premise parameters of the membership function are fixed, the output of ANFIS is obtained. After the training is completed and the results are satisfactory, the FIS parameters and membership function are saved. Fig. 6 illustrates the neurofuzzy module of our proposed method. 


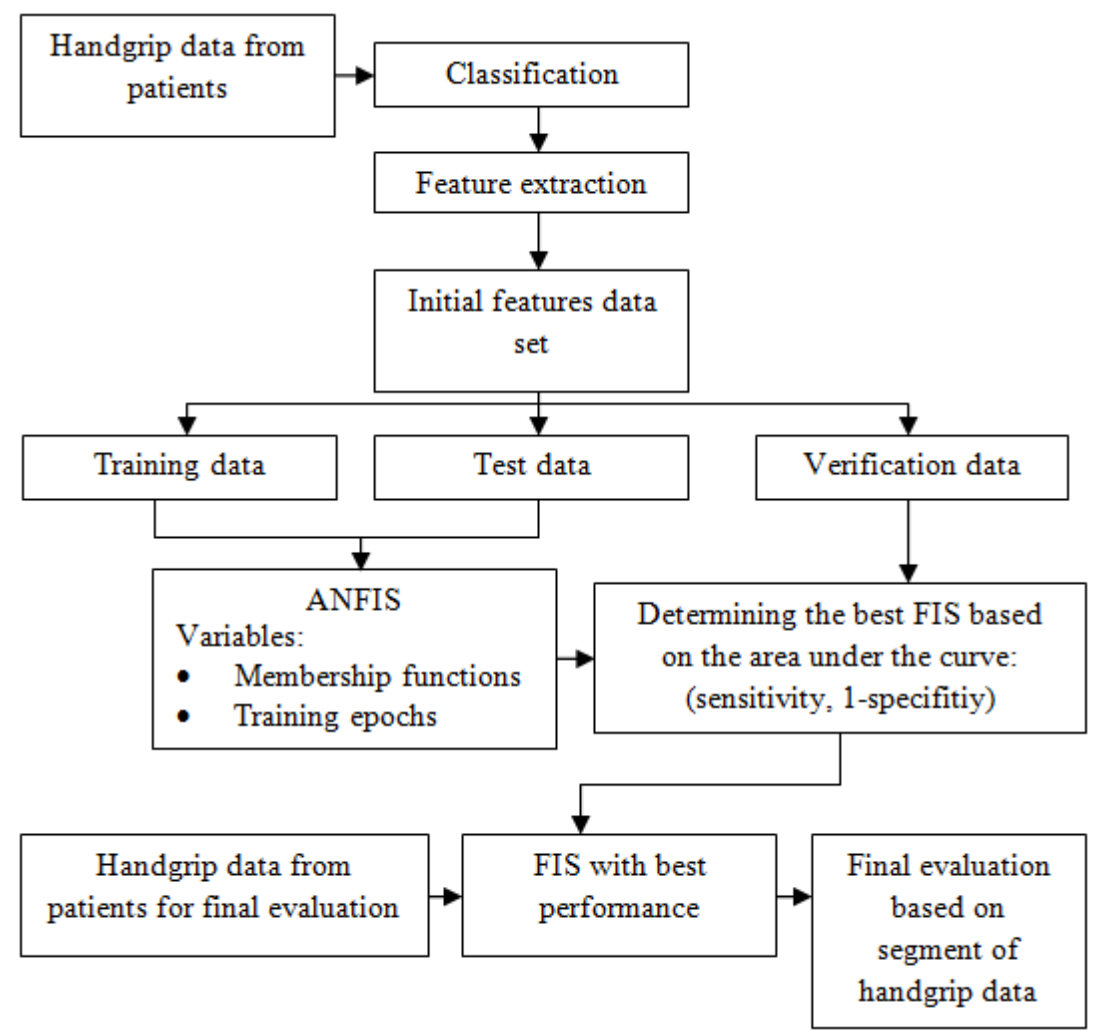

Fig. 6 The Neuro-Fuzzy Module of the Proposed Method

\section{EXPERIMENTAL RESULTS}

After the set of 600 data is obtained from the device, they are analyzed, filtered and classified. The set of 420 data have been randomly chosen to verify the proposed method. The data were pre-classified with the physician to indicate whether it is normal or pathological, followed by recording a field with the value of 0 for pathological or 1 for normal. These records are divided into three groups of training, testing and verification data. The data were trained 7000 epochs to generate FIS. If FIS is not reliable enough, then the FIS is tuned by adding value to the epochs. In ANFIS, the optimization of membership functions performs with minimum error. The error can be reduced by introducing more membership function or by increasing the training epochs. Here is a tradeoff between training time and membership function suitability. Therefore, whenever the result is satisfactory, the FIS performance can be tested. If testing the data and the result is satisfactory, then the FIS parameters and membership functions are saved. Subsequently, the trained ANFIS is verified with pre-classified data. Table 1 shows the accuracy for each normal or pathological patient. The result in classification of the handgrip strength data is quite promising. Even though the classification accuracy looks like biased towards the sample size but the percentage look promising.

Table 1: The Classification Accuracy of Patients

\begin{tabular}{|l|c|c|c|}
\hline \multicolumn{1}{|c|}{ Patient } & Actual & Classification & Accuracy \\
\hline Normal & 300 & 270 & $90 \%$ \\
\hline Pathological & 120 & 90 & $75 \%$ \\
\hline
\end{tabular}


Moreover, the mechanical devise has evolved over time. In order to get the proper reading and correct force value for every handgrip strength value, the devise and the system have to be calibrated. Several weights were loaded to the handgrip, and then the force value recorded. This simple test has been done for five sequence days to get a reliable result; the accuracy of these five-day tests has the tolerance of 0.014 . Table 2 shows the weight and corresponding voltage value. Finally, zeroing-baseline is calculated for the value of hand gripper when there is no weight on it. After that, every reading is deducted by a value of the hand gripper.

From the obtained data shown in Table 2, the following equation is acquired:

$$
y=0.199 x+0.037
$$

Where $y$ is voltage and $x$ is weight. This equation shows the human grip strength value to be used by the software. The algorithm for calculating human handgrip force was built inside the software that is based on the equation stated above. Fig. 7 shows the linear graph of the provided data in Table 2.

Table 2: Force Value for Different Standard Weight

\begin{tabular}{|c|c|c|c|c|c|}
\hline Weight (kg) & 1 & 2 & 3 & 4 & 5 \\
\hline Value (volt) & 0.23 & 0.44 & 0.62 & 0.87 & 1.01 \\
\hline
\end{tabular}

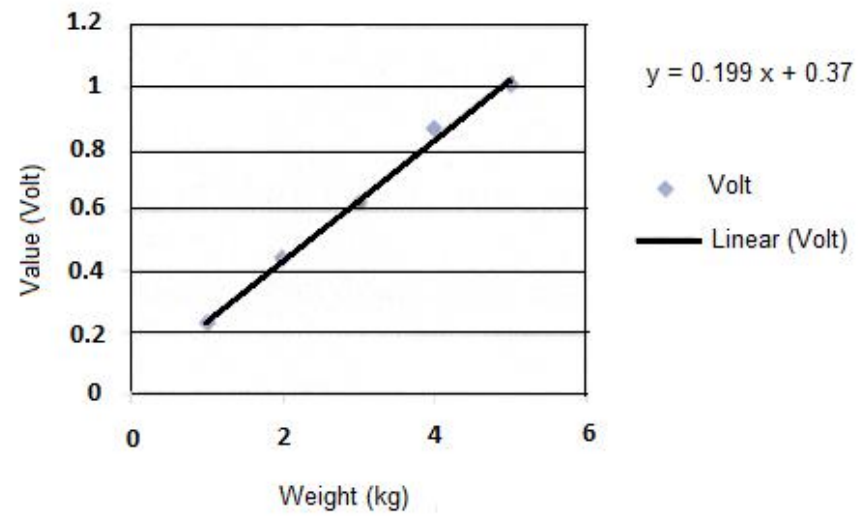

Fig. 7 The Graph of Provided Data in Table 2

\section{DISCUSSION}

This research has resulted in a novel technique of handgrip tension measurements using a neurofuzzy algorithm. Not only this instrument is portable, it is also cost effective. Moreover, it enables more elaborative processing of data resulting in information which could bring better treatment planning, diagnosis and for prognosis study. The LIDO machine [1] has been used previously as handgrip assessment at University Malaya Medical Center (UMMC); it is shown in Fig. 8. The LIDO has been helped and assessed patient's handgrip strength. It only allows the data to be displayed and no automatic storing or any further processing was supported. Its function was also fixed and cannot be changed easily for further needs. Moreover, because this machine is massive, the patients have problem to walk 
near it to be examined. The machine does not have the portability feature as well. The LIDO is the de facto standard for handgrip tension measurement. The graph in Fig. 9 shows the force measurements using the conventional LIDO machine and the result of proposed system using the data of 49 patients. The variance between LIDO and our designed Handgrip is 0.169 for 49 patients. The small variance proves that the proposed system is accurate, reliable and feasible.

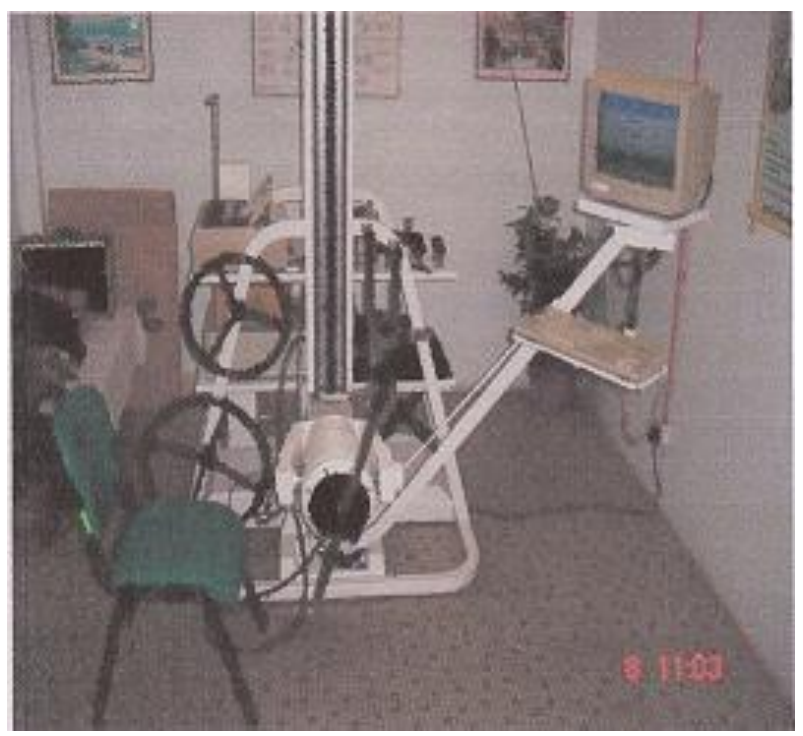

Fig. 8 LIDO Workstation

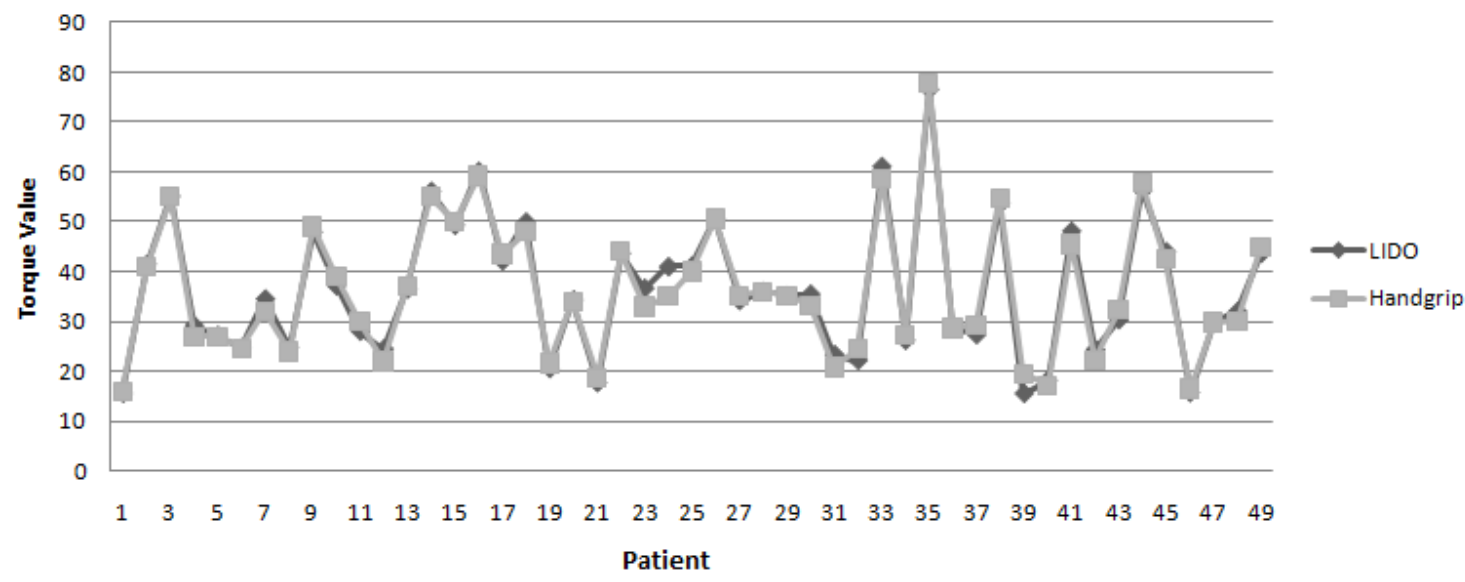

Fig 9 Comparison between LIDO and our Designed Handgrip

The proposed handgrip assessment devise has shown promising result but there are still some problems in the proposed method. The problems are often confronted in neurofuzzy modeling. First, it is about how to adequately decide the number of fuzzy rules extracted from a set of input-output data. Second, it is about how to precisely define the membership functions of each fuzzy rule to solve the problem. If more membership functions of the system is introduced, then it reduces the error.

\section{CONCLUSION AND FUTURE WORK}

Handgrip system is the combination of hardware and software, which embedded with neurofuzzy application. The hardware component includes the mechanical development and the electronic development parts. It consists of the gripper, strain detector and electronic circuit. In software development, the signal analysis includes filtering, 
averaging, amplification and recording the data. Besides the mechanical design of hand gripper, the novelty of the research is the incorporation of Artificial Intelligence (AI) technique for capturing patterns, features, classification and categorization of complex data. It is feasible to extract useful information and classify them using neural fuzzy method. The results are promising, and it is near to human decisions. The obtained result has been compared with result of the LIDO machine. The variance between the LIDO and designed handgrip is 0.169 . The small variance proves that the proposed system is accurate, reliable and feasible. Moreover, the prototype handgrip system provides information of the handgrip tension, and it is possible to make decision of whether the reading indicates normal or pathological patient. The classification accuracy of normal and pathological patients are $90 \%$ and $75 \%$ respectively. Reading and prediction using neurofuzzy algorithm will result in more accurate diagnosis, better treatment planning and more precise prognosis of a patient condition.

As mentioned in the discussion section, the proposed handgrip assessment method has some problems. Future work will be improving the proposed neurofuzzy model. We must find a way to decide the adequate number of fuzzy rules extracted from a set of input-output data. Another future work involves finding an optimal way to discover the sufficient number of membership functions for each fuzzy rule.

\section{Acknowledgment}

We would like to thank Ahmad Hafiz for doing this work as his thesis and implementing the application in a user friendly way. We would also like express our utmost appreciation to the late Prof. Selvanathan who initiated the idea. In addition, we would like to thank Assoc. Prof. Dr. Tunku Kamarul Zaman and Dr. Lydia from Department of Orthopedic at Medical Faculty of University of Malaya for their help and idea sharing.

\section{REFERENCES}

[1] Härkönen, R., Harju R., and Alaranta H. 1993. Acuracy of the JAMAR dynamometer. Journal of Hand Therapy $6(4): 259-262$.

[2] Bear-Lehman, Jane, Patricia A. Miller, Marlo Adler, Jennifer Murray Buonocore, Nancy Coles, Barbara Sweeney Kneafsey, Faygie Katz-Sillman, and Hindy Sherman-Amsel. 2003. An Exploration of Hand Strength and Sensation in Community Elders. Topics in Geriatric Rehabilitation 19 (2):127-136.

[3] Bohannon, R.W. 2001. Dynamometer measurements of hand-grip strength predict multiple outcomes. Perceptual and Motor Skills 93:323-328.

[4] Hafiz, A., S. Anandan, and T. Kamarul. 2005. Development of computerized hand grip strength assessment tool. ASEAN Virtual Instrumentation Applications Contest Submission.

[5] Hafiz, A., N. Selavanthan, S. Anandan, and T. Kamarul. 2005. Hand grip strength assessment tools: A neurofuzzy approach. The International Conference of Biomedical Engineering.

[6] Hafiz, A. 2006. Neuro Fuzzy Analysis on Hand Grip Strength Assessment. University of Malaya, Kuala Lumpur, Malaysia.

[7] Dash, P.K. , A.C. Liew, S. Rahman, and S. Dash. 1995. Fuzzy and neuro-fuzzy computing models for electric load forecasting. Engineering Application of Artificial Intelligence 8 (4):423-433.

[8] Zadeh, L. A. 1965. Fuzzy sets. Information and Control 8 (3):338-353.

[9] Dimitrov, V. Introduction to social fuzziology. http://www.zulenet.com/vladimirdimitrov/pages/IntroSocialFuzz.html. Accessed 3 June 2009.

[10] Jang, J. R., and C. T Sun. 1995. Neuro-fuzzy modeling and control. Proceeding of IEEE 83 (3):378-406. 
[11] Yie-Chien, Chen, and Teng Ching-Cheng. 1995. A model reference control structure using a fuzzy neural network. Fuzzy Sets Syst. 73 (3):291-312. DOI:http://dx.doi.org/10.1016/0165-0114(94)00319-3.

[12] Stergiou, C., and D. Siganos. Neural Network. http://www.doc.ic.ac.uk/ nd/surprise_96/journal/vol4/cs11/report.html. Accessed 3 March 2009.

[13] Y1lmaz, I., and A. G. Yuksek. 2008. An Example of Artificial Neural Network (ANN) Application for Indirect Estimation of Rock Parameters. Rock Mechanics and Rock Engineering 41 (5):781-795.

[14] Lin, C.J., and C.T. Lin. 1996. Reinforcement learning for an ART-based fuzzy adaptive learning control networks. IEEE Transaction on Neural Network 7 (3):709-731.

[15] McGorry, Raymond W. 2001. A system for the measurement of grip forces and applied moments during hand tool use. Applied Ergonomics 32 (3):271-279.

[16] Hoozemans, Marco J. M., and Jaap H. van Dieën. 2005. Prediction of handgrip forces using surface EMG of forearm muscles. Journal of Electromyography and Kinesiology 15 (4):358-366.

\section{BIOGRAPHY}

Woo Chaw Seng is a senior lecturer at the Faculty of Computer Science and Information Technology, University of Malaya. His research interests include image processing and mobile applications.

Mahsa Chitsaz received her B.S. in Computer Engineering from Shiraz University, Iran in 2006. She then obtained her Master of Computer Science from University of Malaya in 2010 under the guidance of Woo Chaw Seng in the area of reinforcement learning in medical image segmentation. 